\title{
Regulation of raft architecture
}

\author{
T. Harder ${ }^{1 *}$ and P. Scheiffele ${ }^{2}$ \\ ${ }^{1}$ Basel Institute for Immunology, Basel and ${ }^{2}$ Department of Molecular and Cell Biology, University of California at Berkeley, California
}

Received April 19, 1999

Accepted May 11, 1999

\begin{abstract}
Summary. Raft membrane domains are envisioned as lateral assemblies of cholesterol and sphingolipids which adopt a liquid-ordered membrane phase. Our understanding of the raft architecture in cell membranes is developing rapidly. The current view describes raft domains as small and highly dynamic subdomains of cell membranes. The size and stability of raft domains are essential parameters which determine the function of raft domains in cells. Here we discuss how the architecture and stability of raft domains is regulated by oligomerisation of raft components and by modulation of their molecular composition.
\end{abstract}

Keywords: Membrane raft; Signal transduction; Sphingolipid; Cholesterol; Oligomerisation.

Abbreviations: DIGs detergent-insoluble glycolipid-enriched membranes; GPI glycosylphosphatidylinositol; PDZ PSD95/discslarge/ZO-1; PI phosphoinositide; $\mathrm{PIP}_{2}$ phosphatidylinositol 4,5bisphosphate; TCR T-cell receptor.

\section{Introduction}

According to the raft hypothesis lateral assemblies of sphingolipids and cholesterol form lipid-based domains (rafts) within biological membranes floating in an environment of glycerophospholipids. Raft domains concentrate specific proteins while excluding others. Following this principle rafts are thought to serve as platforms for the assembly of signalling complexes, the generation of transport vesicles in polarised membrane transport, and the formation of cell-cell or cell-substratum adhesion sites (Brown and London 1998, Simons and Ikonen 1997). In this review we discuss the mechanisms which may regulate raft size and composition and why these features of rafts may be central to their function.

\footnotetext{
* Correspondence and reprints: Basel Institute for Immunology, Grenzacherstrasse 287, CH-4005 Basel, Switzerland.
}

\section{Large detergent-insoluble membrane domains, but small raft domains}

The biophysical nature of raft domains has been studied in artificially reconstituted lipid bilayers. These studies led Brown and colleagues to propose that raft membrane domains exist in a liquid-ordered $\left(l_{0}\right)$ phase, whereas the surrounding nonraft membrane forms a liquid-disordered $\left(l_{d}\right)$ phase (Brown and London 1997). In lipid vesicles which mimic the composition of the plasma membrane, coexistence of these two liquid membrane phases was demonstrated. Phase separation in these membranes depends on cholesterol; and, importantly, model membranes which adopt an $l_{0}$ phase as well as glycosylphosphatidylinositol (GPI)-anchored proteins reconstituted into such membranes are insoluble in mild detergents (Ahmed et al. 1997, Schroeder et al. 1994).

Making use of this property a detergent-insoluble membrane fraction (DIG) isolated from cell membranes is used to analyse the molecular composition of raft domains. DIGs are highly enriched in sphingolipids and cholesterol and contain a specific subset of membrane proteins (Simons and Ikonen 1997). A prominent protein class in the DIG fraction are GPIanchored proteins. DIG association of transmembrane proteins is determined by specific amino acids in their membrane-spanning domains (Scheiffele et al. 1997). Moreover, palmitoylations which anchor cytoplasmic domains of transmembrane proteins to the inner leaflet of the lipid bilayer support DIG association (Melkonian et al. 1999). Several cytoplasmic peripheral membrane proteins, for example Src-kinase family members, are targeted to the DIG fraction via multiple palmitoylation and are thus thought to interact 
with the cytoplasmic face of raft membranes (Rodgers et al. 1994, Shenoy-Scaria et al. 1994).

Even though detergent insolubility has proven a valuable tool and technically easy approach to assay both protein and lipid requirements for raft association, it has its problems. Detergent extraction causes coaggregation of raft markers on the surface of mammalian cells, and the morphology of the DIG membranes does not reflect the organisation of rafts in vivo (Mayor et al. 1994). In order to understand the biological function of rafts, however, their organisation in cell membranes, i.e., their size and composition, needs to be unravelled.

Several recent studies using novel biochemical and biophysical methods analysed raft morphology in the plasma membrane of living cells. Varma and Major (1998) introduced a noninvasive measurement of the distance between GPI-anchored folate receptor molecules. Energy transfer between GPI-anchored folate receptor was monitored by anisotropy measurement of the emitted light. Importantly, energy transfer was independent of the concentration of the folate receptor in the plasma membrane, indicating that folate receptors are clustered in lipid microdomains. These studies, moreover, demonstrated that clustering was cholesterol dependent in line with the role of cholesterol in formation of an $1_{o}$ phase in model membranes. These domains, however, were not resolvable by conventional light microscopy, indicating their small size. Similarily, analysis of GPI-anchored greenfluorescent-protein mutants on the cell surface indicated some degree of clustering (De Angelis et al. 1998). Furthermore, it was demonstrated biochemically that crosslinking efficiencies between GPIanchored proteins were independent of their concentration on the cell surface (Friedrichson and Kurzchalia 1998). Altogether these papers strongly indicate that GPI-anchored proteins on the surface of resting cells are clustered in small domains $(<70 \mathrm{~nm}$ diameter) with dimensions below the resolution limit of conventional light microscopy.

\section{Fission and fusion of raft domains - a functional switch?}

What is the functional significance of these raft microclusters? Such small raft domains can harbour only relatively few protein molecules. Moreover, it appears improbable that membrane domains of this size can be shaped into transport vesicles or function as platforms for signal transduction. However, assuming a regulated equilibrium of fusion and fission of raft domains in the cell surface the localisation of a molecule in rafts would have important functional consequences.

In the resting state there is a large number of domains which are dispersed over the cell surface. The molecules in the raft domain would only have limited contact with molecules in the surrounding "nonraft" membrane. For signal transduction molecules this could mean that unwanted cross talk is prevented. As the domains are small, the local representation of signalling compounds will not be sufficient to recruit downstream binding partners.

When rafts coalesce, molecules previously separated by dispersion of the raft domains are combined in a common large patch (for detailed discussion, see Vaz and Almeida 1993). Furthermore, now large domains with high local concentrations of raft components are formed. These concentrations would now be above the threshold to tightly bind downstream molecules (Fig. 1).

Different mechanisms should allow to shift the equilibrium of raft size. The availability of raft lipids or the lipid composition in a membrane as well as extracellular or intracellular cross-linking events and their influence on raft architecture will be discussed below.

\section{Regulation of rafts by changes in lipid composition}

The amount of raft lipids in a membrane should be an important parameter for the size of the domains. According to the proposed equilibrium of raft fusion and fission, increase of a raft lipid should shift this equilibrium towards the fusion and decrease of raft lipids towards the fission of the domains. The endoplasmic reticulum membrane might represent an extreme case for fission as it contains cholesterol but only minute amounts of sphingolipids. No DIG association of proteins is observed in the endoplasmic reticulum membrane, suggesting that the sphingolipid levels are below the threshold to significantly assemble rafts (Brown and Rose 1992).

Relatively little is known how physiological changes in sphingolipid composition change the properties of raft domains. Such changes in glycolipid expression have been described for cell differentiation processes. For example, maturation of neurons to form a fully polarised phenotype is accompanied by an upregulation of specific glycolipids (Futerman et al. 1998). 


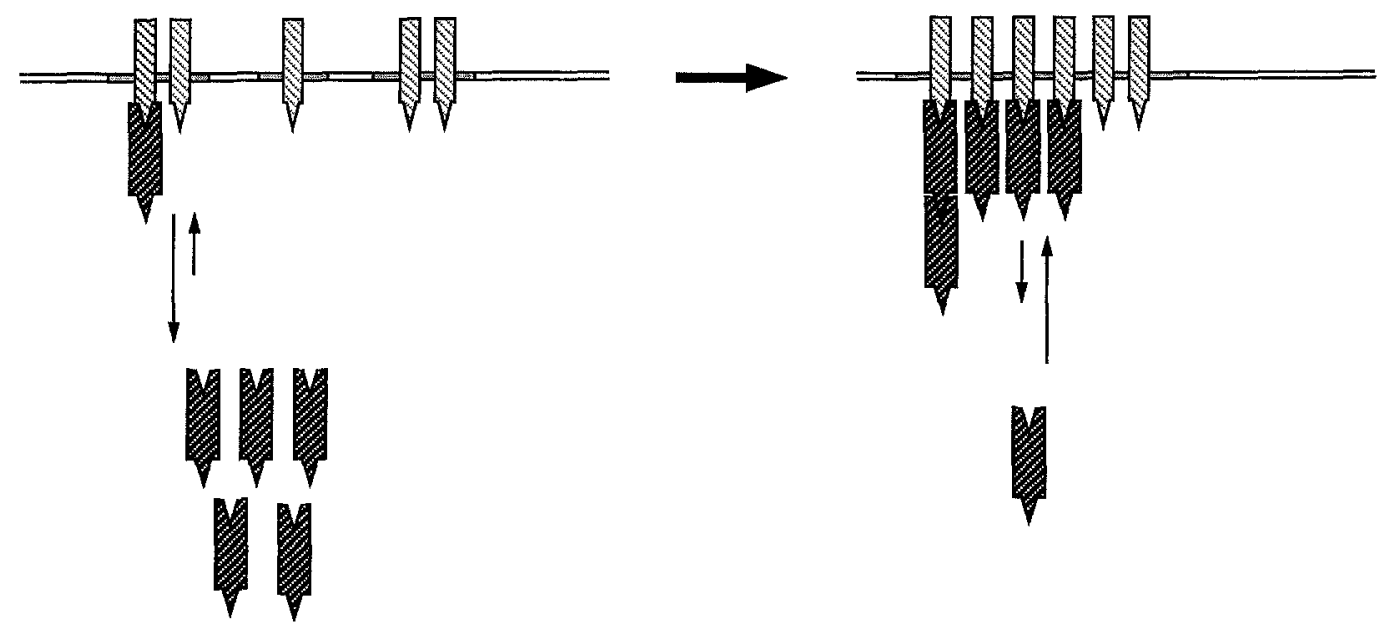

Fig. 1. Recruitment of cytosolic proteins to stabilised raft domains triggered by clustering. Clustering of a raft-associated membrane protein creates large patches with a high local concentration of this membrane protein. This allows efficient recruitment of a cytosolic ligand which binds only weakly to this membrane protein under steady-state conditions. Multiple binding sites or lateral interaction between the ligand molecules may lead to a high cooperativity in the association

Interestingly, this upregulation correlates with the stability of detergent-insoluble complexes and sorting of membrane proteins to the axon (Ledesma et al. 1999). Similarly, the amount of ganglioside GM1 in resting $\mathrm{T}$ lymphocytes strongly increases after priming by engagement of T-cell receptor (TCR) and costimulation. This priming induces differentiation of the resting $T$ lymphocyte towards an effector $T$ cell. Upregulation of the glycolipid GM1 may drive raft fusion and thereby increase responsiveness of the cells (A. Viola and T. Harder unpubl. results). It will be most interesting to see which other lipid components are upregulated in such maturation processes, as one would expect that for example also the cholesterol pool in the plasma membrane increases.

Besides sphingolipids and cholesterol, phosphoinositides (PI) such as phosphatidylinositol-4,5-bisphosphate $\left(\mathrm{PIP}_{2}\right)$ are highly enriched in the DIG fraction (Hope and Pike 1996). PI are rapidly turned over by the activity of PI kinases and their respective counteracting phosphatases (Liu et al. 1998) and may be subject to short-term changes in rafts. PI kinases and phosphatases may therefore play an essential role in regulating raft composition. Downstream targets which bind $\mathrm{PIP}_{2}$, such as cytoskeletal proteins or signalling molecules, could then aid the assembly of the raft patches and stabilise these domains (Fukami et al. 1994). Furthermore $\mathrm{PIP}_{2}$ is rapidly hydrolysed in activated cells by the activity of phospholipase $C$, yielding second messengers inositol 1,4,5-trisphosphate and diacylglycerol. This hydrolysis may be a mechanism of local raft destabilisation and downregulation of its activity (Pike and Casey 1996, Stauffer et al. 1998).

\section{Clustering rafts from within the membrane: caveolin}

Members of the caveolin family were initially described as major constituent of the caveolar invaginations in the plasma membrane and the DIG fraction. A number of features make caveolin an ideal candidate for sequestering raft domains. The protein tightly binds to cholesterol, approximately in equimolar stoichiometry. Furthermore, each caveolin molecule is modified by three palmitic acid chains in the C-terminal cytoplasmic domain. Caveolins form large oligomers of 15-20 molecules, which might allow creation of large stabilised rafts within the membrane. Consistently, the ganglioside GM1 is found concentrated in cell surface caveolae (for a review, see Parton 1996). A multitude of biological functions have been assigned to caveolae. The common theme in all these processes might be the organisation of a specific membrane subcompartment. Among these functions are cholesterol exchange, endocytosis and transcytosis of compounds in endothelia, formation of signalling centres, etc. (Okamoto et al. 1998).

However, caveolins were also observed in noncaveolar regions of the cell surface and in intracellular compartments (Dupree et al. 1993). Different complexes of caveolins are involved in polarised mem- 
brane sorting from the trans-Golgi network in MDCK cells. Apical transport appears to involve caveolin-1 homo-oligomers, whereas basolateral transport vesicles contain complexes of caveolin-1 and -2 (Scheiffele et al. 1998). The apical complexes show a significant size shift upon leaving the Golgi complex, possibly reflecting the specific concentration of apical protein and lipid cargo. Interestingely caveolin was shown to be required for the formation of active signalling complexes of integrins, Fyn kinase, and associating signalling molecules (Wary et al. 1998). Future studies will show to which extent caveolins function in these complexes by coordinating protein-protein or protein-lipid interactions.

\section{Extracellular cross-linking: oligomeric ligands of raft-associated receptors}

Many ligands induce clustering of their receptors upon binding. Additionally, repetitive antigens and other oligomeric molecules can cluster their cognate cell surface receptors, and this may induce formation of stabilised raft domains. One of the first examples described is the cross-linking of the immunoglobulin $E$ receptor by repetitive antigens via receptor-bound immunoglobulin $E$ molecules (Holowka and Baird 1996). These domains are preferentially stained with specific lipid dyes and moreover transiently accumulate the glycosphingolipid GM1 (Stauffer and Meyer 1997, Thomas et al. 1994). Extracellular cross-linking of membrane proteins is a frequently recurring theme in the interaction of cells with their environment. For example, membrane protein clusters are formed when cell receptors interact with extracellular matrix or when cell adhesion molecules line up at cell-cell contact sites. It is tempting to speculate that some of these clusters have raft properties which are recognised by an intracellular machinery.

\section{Intracellular cross-linking: adaptor cross-linking}

Also cytoplasmic molecules can confer clustering of transmembrane receptors. For example PDZ (PSD-95, discs-large, ZO-1) domain-containing proteins are found at different cellular junctions like synapses and adherens junctions in the epithelium. Via the PDZ domain they bind to a recognition sequence located at the $\mathrm{C}$ terminus of their ligands. One of the most prominent members of this family, PSD-95, was recently reported to be recovered in DIGs (Perez and Bredt 1998). Surprisingly, DIG association in heterologous cells did not require the two $\mathrm{N}$-terminal palmitoylations of PSD-95 but the PDZ domain-containing region. However, targeting of PSD-95 to synaptic clusters in primary neurons was dependent on the palmitoylation (Craven et al. 1999). Possibly, in heterologous cells binding to a raft-associated transmembrane protein mediates recruitment into DIGs as it was observed for another PDZ domain-containing protein, GRIP2. Via one of its seven PDZ domains GRIP2 interacts with the $\mathrm{C}$ terminus of EphrinB1, a transmembrane ligand of Eph-tyrosine kinase receptors, and is thereby recruited into rafts (Brückner et al. 1999). Binding of transmitter receptors or channels to PSD-95 results in clustering in the membrane which is driven by oligomerisation of PSD-95 (Hsueh et al. 1997). It will be interesting to know whether these patches formed by PSD-95 at the synapse represent raft domains.

A series of recent reports suggest that many proteins involved in TCR-mediated signalling are associated to rafts. These include the phosphorylated $\zeta$-subunit of the CD3-TCR complex itself, the Src kinase family members Fyn and Lck, the adaptor protein LAT (linker for activation of T lymphocytes), and a large number of other molecules involved in T-lymphocyte activation (Montixi et al. 1998, Xavier et al. 1998, Zhang et al. 1998). For LAT and Lck a link between their DIG association and their capability to transduce a signal via TCR was described (Kabouridis et al. 1997, Rodgers et al. 1994, Shenoy-Scaria et al. 1994, Zhang et al. 1998).

Under physiological conditions antigen-presenting cells display only few peptide-major histocompatibility complexes specific for a TCR of a particular T cell. Thus, triggering of $T$ lymphocytes most probably does not involve the formation of large TCR clusters. Rather it appears that cognate major histocompatibility molecules can serially trigger multiple rounds of TCRs until a threshold for activation is reached (Valitutti et al. 1995b, Viola and Lanzavecchia 1996). A series of recent reports underline the importance of raft domain sequesteration and stabilisation in the $T$ lymphocyte's plasma membrane region contacting the antigen-presenting cell. Firstly raft-associated glycosphingolipid GM1 accumulates at contact sites between a naive $\mathrm{T}$ lymphocyte and a TCR-activating bead in a manner depending on costimulation by 
CD28 engagement (Viola et al. 1999). Moreover coengagement of TCR and the GPI-anchored surface protein CD48 enhanced T-cell activation and Triton X100 insolubility of TCR subunits (Moran and Miceli 1998). A multitude of additional interactions between accessory molecules and ligands support the formation of a contact zone between an antigen-presenting cell and a $T$ cell. This zone adopts a well defined structure which may represent a highly differentiated scaffold for raft patches involved in the activation of $\mathrm{T}$ cell by antigen-presenting cells (Dustin and Shaw 1999, Monks et al. 1998, Moran and Miceli 1998).

It appears probable that intracellular proteins are responsible for accumulating and stabilising membane domains at the site of TCR engagement. Cross-linking of raft-associated molecules could occur through multiple interactions of adaptor molecules which generate a complex network of signalling molecules sufficient to stabilise raft domains. Most probably the actin cytoskeleton plays an important role in this assembly process. Signalling via TCR requires an intact actin cytoskeleton (Valitutti et al. 1995a). Moreover, it was shown that artificially induced clusters of the glycosphingolipid GM1 accumulate phosphotyrosine as well as actin filaments (Harder and Simons 1999). The actin cytoskeleton may help organise the T-cell contact site via an active actomyosin-mediated transport of molecules and/or by forming a scaffold for raft domains (Dustin and Shaw 1999, Kupfer et al. 1987, Wülfing and Davis 1998).

Rafts, actin cytoskeleton or other scaffolding proteins, and signalling molecules are likely to be tied up in an interplay as presented in Fig. 2. Triggering of receptors may induce the recruitment of proteins which aid raft sequestration. Raft-associated $\mathrm{PIP}_{2}$ may induce actin polymerisation and recruit a set of cytoskeletal molecules to the rafts, which could form a scaffold for further stabilisation of the domains (Fukami et al. 1994). Moreover, signalling may induce actin polymerisation for example via activation of GTPases of the Rho family (Reif and Cantrell 1998). Large raft patches could then serve as a platform for assembly of activated signalling complexes. This positive feedback circuit may ensure that a threshold for a cellular response is reached even if few signalling receptors are triggered. It is the challenge for the future to elucidate how a starting point for raft stabilisation is seeded and which mechanisms mediate and regulate the ongoing stabilisation.
Trigger

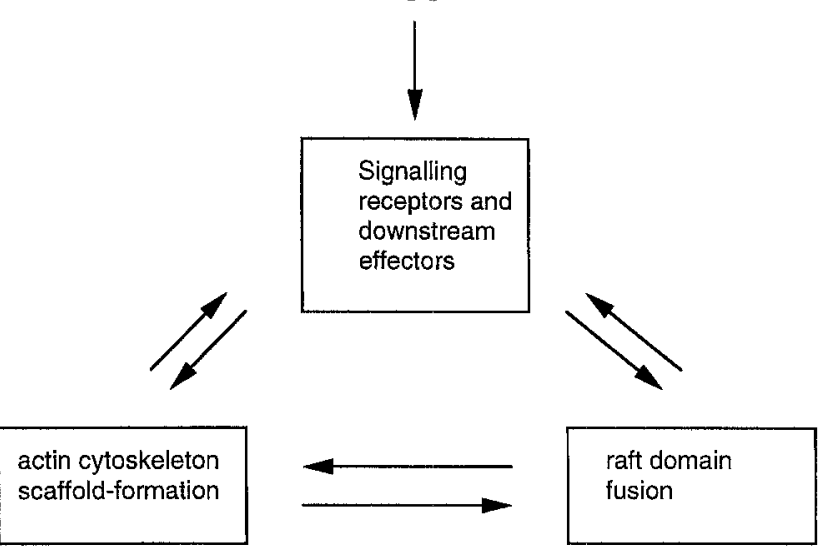

Fig. 2. Possible interplay of signalling molecules, raft domains, and cytoskeletal elements. Membrane receptors are triggered by binding of ligands. This may induce fusion and accumulation of raft domains either by cross-linking of receptors via the ligands or by recruitment of intracellular signalling molecules to the membrane. Further recruitment of raft-associated signalling molecules and actin accumulation allows stabilisation of the raft patches. Once overcoming the initial threshold for the formation of a large raft patch, a positive feedback cycle may serve to amplify signalling via the raftassociated receptors

\section{Acknowledgments}

We thank Christian Wimmer and Antonella Viola for critical reading of the manuscript. The Basel Institute for Immunology was founded and is supported by Hoffman La Roche Ltd. P.S. is supported by an EMBO long-term fellowship.

\section{References}

Ahmed SN, Brown DA, London E (1997) On the origin of sphingolipid/cholesterol-rich detergent-insoluble cell membranes: physiological concentrations of cholesterol and sphingolipid induce formation of a detergent-insoluble, liquid-ordered lipid phase in model membranes. Biochemistry 36: 10944 10953

Brown DA, London E (1997) Structure of detergent-resistant membrane domains: does phase separation occur in biological membranes? Biochem Biophys Res Commun 240: 1-7

- - (1998) Functions of lipid rafts in biological membranes. Annu Rev Cell Dev Biol 14: 111-136

- Rose JK (1992) Sorting of GPI-anchored proteins to glycolipidenriched membrane subdomains during transport to the apical cell surface. Cell 68: 533-544

Brückner K, Labrador JP, Scheiffele P, Merbst A, Seeburg PH, Klein R (1999) EphrinB ligands recruit GRIP family PDZ adaptor proteins into raft membrane microdomains. Neuron 22 : 511-524

Craven SE, El-Husseini AE, Bredt DS (1999) Synaptic targeting of the postsynaptic density protein PSD-95 mediated by lipid and protein motifs. Neuron 22: 497-509 
De Angelis DA, Miesenbock G, Zemelman BV, Rothman JE (1998) PRIM: proximity imaging of green fluorescent protein-tagged polypeptides. Proc Natl Acad Sci USA 95: 12312-12316

Dupree P, Parton RG, Raposo G, Kurzchalia TV, Simons K (1993) Caveolae and sorting in the trans-Golgi network of epithelial cells. EMBO J 1993 12: 1597-1605

Dustin ML, Shaw AS (1999) Costimulation: building an immunological synapse. Science 283: 649-650

Friedrichson T, Kurzchalia TV (1998) Microdomains of GPIanchored proteins in living cells revealed by crosslinking. Nature 394: 802-805

Fukami K, Endo T, Imamura M, Takenawa T (1994) Alpha-actinin and vinculin are PIP2-binding proteins involved in signaling by tyrosine kinase. J Biol Chem 269: 1518-1522

Futerman AH, Boldin S, Brann AB, Schwarz A, Zisling R (1998) Regulatory roles for sphingolipids in the growth of polarized neurons. Ann N Y Acad Sci 845: 176-187

Harder T, Simons K (1999) Clusters of glycolipid and glycosylphosphatidylinositol-anchored proteins in lymphoid cells: accumulation of actin regulated by local tyrosine phosphorylation. Eur $\mathrm{J}$ Immunol 29: 556-562

Holowka D, Baird B (1996) Antigen-mediated IGE receptor aggregation and signaling: a window on cell surface structure and dynamics. Annu Rev Biophys Biomol Struct 25: 79-112

Hope HR, Pike LJ (1996) Phosphoinositides and phosphoinositideutilizing enzymes in detergent-insoluble lipid domains. Mol Biol Cell $7: 843-851$

Hsueh YP, Kim E, Sheng M (1997) Disulfide-linked head-to-head multimerization in the mechanism of ion channel clustering by PSD-95. Neuron 18: 803-814

Kabouridis PS, Magee AI, Ley SC (1997) S-acylation of LCK protein tyrosine kinase is essential for its signalling function in $\mathrm{T}$ lymphocytes. EMBO J 16: 4983-4998

Kupfer A, Swain SL, Singer SJ (1987) The specific direct interaction of helper T cells and antigen-presenting B cells II: reorientation of the microtubule organizing center and reorganization of the membrane-associated cytoskeleton inside the bound helper $\mathrm{T}$ cells. J Exp Med 165: 1565-1580

Ledesma MD, Brugger B, Bünning C, Wieland FT, Dotti CG (1999) Maturation of the axonal plasma membrane requires upregulation of sphingomyelin synthesis and formation of protein-lipid complexes. EMBO J 18: 1761-1771

Liu Y, Casey L, Pike LJ (1998) Compartmentalization of phosphatidylinositol 4,5-bisphosphate in low-density membrane domains in the absence of caveolin. Biochem Biophys Res Commun 245: 684-690

Mayor S, Rothberg KG, Maxfield FR (1994) Sequestration of GPIanchored proteins in caveolae triggered by cross-linking. Science 264: 1948-1951

Melkonian KA, Ostermeyer AG, Chen JZ, Roth MG, Brown DA (1999) Role of lipid modifications in targeting proteins to detergent-resistant membrane rafts: many raft proteins are acylated, while few are prenylated. J Biol Chem 274: 3910-3917

Monks CR, Freiberg BA, Kupfer H, Sciaky N, Kupfer A (1998) Three-dimensional segregation of supramolecular activation clusters in T cells. Nature $395: 82-86$

Montixi C, Langlet C, Bernard AM, Thimonier J, Dubois C, Wurbel MA, Chauvin JP, Pierres M, He HT (1998) Engagement of $\mathrm{T}$ cell receptor triggers its recruitment to low-density detergentinsoluble membrane domains. EMBO J 17: 5334-5348

Moran M, Miceli MC (1998) Engagement of GPI-linked CD48 contributes to TCR signals and cytoskeletal reorganization: a role for lipid rafts in T cell activation. Immunity 9: 787-796

Okamoto T, Schlegel A, Scherer PE, Lisanti MP (1998) Caveolins, a family of scaffolding proteins for organizing "preassembled sig- naling complexes" at the plasma membrane. J Biol Chem 273: $5419-5422$

Parton RG (1996) Caveolae and caveolins. Curr Opin Cell Biol 8: $542-548$

Perez AS, Bredt DS (1998) The N-terminal PDZ-containing region of postsynaptic density-95 mediates association with caveolar-like lipid domains. Neurosci Lett 258: 121-123

Pike LJ, Casey L (1996) Localization and turnover of phosphatidylinositol 4,5-bisphosphate in caveolin-enriched membrane domains. J Biol Chem 271: 26453-26456

Reif K, Cantrell DA (1998) Networking Rho family GTPases in lymphocytes. Immunity 8: 395-401

Rodgers W, Crise B, Rose JK (1994) Signals determining protein tyrosine kinase and glycosyl-phosphatidylinositol-anchored protein targeting to a glycolipid-enriched membrane fraction. Mol Cell Biol 14: 5384-5391

Scheiffele P, Roth MG, Simons K (1997) Interaction of influenza virus haemagglutinin with sphingolipid-cholesterol membrane domains via its transmembrane domain. EMBO J 16: 55015508

- Verkade P, Fra AM, Virta H, Simons K, Ikonen E (1998) Caveolin-1 and -2 in the exocytic pathway of MDCK cells. J Cell Biol 140: 795-806

Schroeder R, London E, Brown D (1994) Interactions between saturated acyl chains confer detergent resistance on lipids and glycosylphosphatidylinositol (GPI)-anchored proteins: GPIanchored proteins in liposomes and cells show similar behavior. Proc Natl Acad Sci USA 91: 12130-12134

Shenoy-Scaria AM, Dietzen DJ, Kwong J, Link DC, Lublin DM (1994) Cysteine 3 of Src family protein tyrosine kinase determines palmitoylation and localization in caveolae. J Cell Biol 126 : $353-363$

Simons K, Ikonen E (1997) Functional rafts in cell membranes. Nature 387: 569-572

Stauffer TP, Meyer T (1997) Compartmentalized IgE receptormediated signal transduction in living cells. J Cell Biol 139: 14471454

Ahn S, Meyer T (1998) Receptor-induced transient reduction in plasma membrane PtdIns(4,5)P2 concentration monitored in living cells. Curr Biol 8: 343-346

Thomas JL, Holowka D, Baird B, Webb WW (1994) Large-scale coaggregation of fluorescent lipid probes with cell surface proteins. J Cell Biol 125: 795-802

Valitutti S, Dessing M, Aktories K, Gallati H, Lanzavecchia A (1995a) Sustained signaling leading to $T$ cell activation results from prolonged $\mathrm{T}$ cell receptor occupancy: role of $\mathrm{T}$ cell actin cytoskeleton. J Exp Med 181: 577-584

- Muller S, Cella M, Padovan E, Lanzavecchia A (1995b) Serial triggering of many T-cell receptors by a few peptide-MHC complexes. Nature 375: 148-151

Varma R, Mayor S (1998) GPI-anchored proteins are organized in submicron domains at the cell surface. Nature 394: 798-801

Vaz WL, Almeida PF (1993) Phase topology and percolation in multi-phase bilayers: is the biological membrane a domain mosaic? Curr Opin Struct Biol 3: 482-488

Viola A, Lanzavecchia A (1996) T cell activation determined by $\mathrm{T}$ cell receptor number and tunable thresholds. Science 273: 104106

- Schroeder S, Sakakibara Y, Lanzavecchia A (1999) T lymphocyte costimulation mediated by reorganization of membrane microdomains. Science 283: 680-682

Wary KK, Mariotti A, Zurzolo C, Giancotti FG (1998) A requirement for caveolin-1 and associated kinase Fyn in integrin signaling and anchorage-dependent cell growth. Cell 94: 625634 
Wülfing C, Davis MM (1998) A receptor/cytoskeletal movement triggered by costimulation during $\mathrm{T}$ cell activation. Science 282 : 2266-2269

Xavier R, Brennan T, Li Q, McCormack C, Seed B (1998) Membrane compartmentation is required for efficient $\mathrm{T}$ cell activation. Immunity 8: 723-732
Zhang W, Trible RP, Samelson LE (1998) LAT palmitoylation: its essential role in membrane microdomain targeting and tyrosine phosphorylation during $\mathrm{T}$ cell activation. Immunity 9:239246 